Cahiers
de la Recherche
surles Droits Fondamentaux
Cahiers de la recherche sur les droits fondamentaux

17 | 2019

La motivation des actes administratifs. Le droit français à la lumière du droit administratif comparé

\title{
La motivation des actes administratifs au Canada
}

Daniel Mockle

\section{(2) OpenEdition}

Journals

Édition électronique

URL : https://journals.openedition.org/crdf/3790

DOI : $10.4000 /$ crdf.3790

ISSN : 2264-1246

Éditeur

Presses universitaires de Caen

Édition imprimée

Date de publication : 31 décembre 2019

Pagination : 125-137

ISBN : 978-2-84133-960-0

ISSN : $1634-8842$

Référence électronique

Daniel Mockle, "La motivation des actes administratifs au Canada », Cahiers de la recherche sur les droits fondamentaux [En ligne], 17 | 2019, mis en ligne le 06 février 2021, consulté le 15 novembre 2022. URL : http://journals.openedition.org/crdf/3790 ; DOI : https://doi.org/10.4000/crdf.3790 


\title{
La motivation des actes administratifs au Canada
}

\author{
Daniel MOCKLE \\ Professeur de droit public à la faculté de science politique et de droit de l'université du Québec à Montréal (Canada)
}

I. La prépondérance du contrôle judiciaire pour l'évaluation des exigences de motivation

II. Les exigences de justification en matière réglementaire

III. La nécessité d'un cadre législatif

En dépit de sa proximité géographique avec les ÉtatsUnis, le Canada offre un net contraste sur la question de la motivation des actes administratifs, notamment pour le rôle de la loi dans la systématisation du droit administratif. Contrairement aux orientations retenues en droit américain, le Canada, au niveau du droit fédéral, n'a élaboré aucune loi générale qui pourrait être comparée à l'Administrative Procedure Act (APA) de 1946 avec ses amendements subséquents. Le système canadien est resté fidèle à l'élaboration des règles et principes du droit administratif dans les systèmes de droit issus de la filière britannique où le juge joue un rôle déterminant (judge made law). Ce constat doit néanmoins être nuancé dans la mesure où des tentatives de codification de la procédure administrative ont été proposées, notamment celle suggérée en 1985 par la défunte Commission de réforme du droit pour le fonctionnement des organismes administratifs autonomes ${ }^{1}$, ainsi que celle initiée en 1995 par le ministère de la Justice du Canada et qui n'a connu aucune suite ${ }^{2}$. Faute d'une obligation générale de motivation, ne serait-ce que pour les décisions défavorables, le législateur a parfois reconnu cette nécessité dans des lois plus spécifiques ${ }^{3}$.

Si le droit fédéral n'offre aucun cadre législatif de portée générale en matière de procédure administrative, le Canada n'en reste pas moins un État fédéral où quatre provinces ont élaboré des lois de procédure qui visent, à divers degrés, le fonctionnement des tribunaux administratifs (administrative tribunals), ainsi que des dimensions plus générales de procédure administrative non contentieuse. Si l'Alberta a été la première à formuler des règles procédurales en $1966^{4}$, suivie par l'Ontario en $1993^{5}$, une étape décisive a été franchie par le Québec en $1997^{6}$, suivi sur ce plan par la

1. Commission de réforme du droit du Canada, Les organismes administratifs autonomes / Independant Administrative Agencies, Ottawa, Commission de réforme du droit du Canada (Rapport/Commission de réforme du droit du Canada ; 26), 1985, p. 8o (la motivation des décisions de rejet était prévue). En 1990, l'Association du Barreau canadien a suggéré une réforme du processus de nomination des membres des organismes administratifs sans englober les règles de procédure: Association du Barreau canadien, L'indépendance des tribunaux et organismes administratifs fédéraux au Canada, Ottawa, L'Association, 1990.

2. Ministère de la Justice du Canada, Proposition d'une loi fédérale sur les pouvoirs et la procédure concernant les audiences administratives, 1995 .

3. Loi sur l'immigration et la protection des réfugiés, LC 2001, chap. 27, art. 33 sq. (motifs requis pour prononcer une interdiction de territoire) et art. 57-58 (motifs requis pour la détention); loi sur les produits antiparasitaires, LC 2002, chap. 28, $\$ 17(5)$ (motivation obligatoire pour l'homologation de certains produits); loi sur la radiodiffusion, LC 1991, chap. 11, $\$ 28(5)$ (motivation requise pour le Cabinet afin de modifier les décisions du Conseil de la radiodiffusion et des télécommunications - CRTC); loi sur le système correctionnel et la mise en liberté sous condition, LC 1992, chap. 20, art. 34 (motifs requis pour le maintien d'un détenu en isolement préventif). Voir, en fin d'article, la liste des sigles désignant les recueils de lois.

4. Administrative Procedures and Jurisdiction Act, SA 1996, chap. 1; loi reprise et intégrée dans RSA 2000, chap. A-3.

5. Loi sur l'exercice des compétences légales / Statutory Powers Procedure Act, LO 1993, chap. 27; reprise et intégrée dans LRO 1990 , chap. S-22.

6. Loi sur la justice administrative / Act Respecting Administrative Justice, LRQ, chap. J-3. 
Colombie-Britannique en 20047 . La législation de l'Ontario, ainsi que celle de la Colombie-Britannique, ne visent que les tribunaux administratifs. Le Québec fait clairement la distinction entre l'exercice d'une fonction administrative et celui résultant d'une fonction juridictionnelle. Pour l'exercice d'une fonction administrative, seules les décisions administratives défavorables doivent être motivées, alors qu'en contrepartie, pour l'exercice d'une fonction juridictionnelle, toutes les décisions doivent être écrites et motivées ${ }^{8}$. Le lecteur francophone doit être informé que l'expression «tribunaux administratifs », qui est utilisée au Canada, est l'équivalent fonctionnel pour administrative tribunals, ce qui correspond en France, et dans d'autres pays de la francophonie, à des autorités administratives indépendantes qui sont responsables d'un contentieux spécialisé. Tout en relevant de ce cadre général, le Québec s'en démarque néanmoins avec la création, en 1997, du tribunal administratif du Québec, qui est très proche du modèle des cours judiciaires. Ce tribunal exerce exclusivement des fonctions juridictionnelles afin de recevoir et d'évaluer des requêtes en contestation de décisions administratives ${ }^{9}$.

Afin de clarifier le cadre juridique de la motivation des actes administratifs au Canada, cette différence est importante car, en définitive, elle oppose des actes administratifs et des actes juridictionnels. Ce qui complique davantage la compréhension du droit canadien pour les lecteurs francophones, c'est l'absence de principe d'une théorie générale des actes administratifs. En France, cette catégorie était nommément visée par la loi du 11 juillet 1979 relative à la motivation des actes administratifs et à l'amélioration des relations entre le public et l'administration ${ }^{10}$. Cette approche a été conservée dans le Code des relations entre le public et l'administration de 2015 . Le titre premier du livre II a pour objet explicite «La motivation et la signature des actes administratifs ${ }^{11}$. Ce cadre conceptuel diffère notablement du Canada où le droit administratif repose sur la filiation britannique. Sur le plan culturel et juridique, la tradition de common law reste prépondérante, à l'exception du Québec. Dans les systèmes issus de cette tradition, il n'existe pas une théorie générale des actes administratifs comme dans les systèmes romanistes. Compte tenu de l'existence d'une tradition de droit civil pour les matières de droit privé au Québec, l'évolution du droit administratif y est néanmoins différente, notamment pour l'élaboration de la théorie des actes administratifs ${ }^{12}$. Dès la parution des premiers ouvrages de droit administratif, les auteurs ont cherché à systématiser cette partie du droit administratif sur le modèle offert par les systèmes romanistes.

Dans le premier tome de leur Traité de droit administratif, René Dussault et Louis Borgeat ont élaboré une deuxième partie qui a pour objet «Les actes de l'Administration». Les trois chapitres, qui visent, dans l'ordre de présentation, la décision, le règlement et le contrat, sont précédés d'un prologue de onze pages qui expose des concepts propres à la théorie des actes administratifs ${ }^{13}$. Dans un chapitre relatif à l'action administrative, Patrice Garant rappelle qu'il existe des actes administratifs à caractère proprement administratif, des actes administratifs à caractère législatif (les règlements) et des actes administratifs à caractère juridictionnel (les actes quasi judiciaires) ${ }^{14}$. Dans le troisième chapitre de leur ouvrage qui a pour objet « La théorie des actes administratifs», Pierre Issalys et Denis Lemieux proposent un schéma général qui correspond aux exigences de la théorie des actes administratifs ${ }^{15}$. Si, pour les auteurs francophones, cet effort de systématisation $s^{\prime}$ impose comme une évidence ${ }^{16}$, il en va tout autrement pour les auteurs anglophones qui l'ignorent complètement, dans la continuité de la filière britannique où sont utilisés les termes "fonctions» et "pouvoirs ${ }^{17}$.

Cette différence pourrait être réduite à des dimensions académiques afin de souligner le bijuridisme qui caractérise le droit canadien. Sans pour autant prendre position sur l'existence d'une théorie générale des actes administratifs, la Cour suprême a néanmoins utilisé le terme «acte administratif» afin de préciser la nature juridique des règlements municipaux. Elle a franchi ce pas dans un contexte où la nature juridique du règlement contesté n'était pas en cause ${ }^{18}$. La Cour a ainsi fait

7. Administrative Tribunals Act, SBC 2004, chap. 45 .

8. Loi sur la justice administrative, art. 8 (fonction administrative) et art. 13 (fonction juridictionnelle). L'article 13 dispose que «La décision terminant une affaire doit être écrite et motivée, même si elle a été portée oralement à la connaissance des parties».

9. Ibid., titre II de la loi ( «e Tribunal administratif du Québec»).

10. Loi $\mathrm{n}^{\circ} 79-587$ du 11 juillet 1979 relative à la motivation des actes administratifs et à l'amélioration des relations entre le public et l'administration, Journal officiel de la République française, 12 juillet 1979, p. 1711.

11. Code des relations entre le public et l'administration, ordonnance $\mathrm{n}^{\circ} 2015-1341$ du 23 octobre 2015, Journal officiel de la République française, 25 octobre 2015, p. 19872.

12. D. Mockle, «De quelques influences croisées dans l'élaboration de la théorie des actes administratifs", in Vastes mondes. Études en l'honneur du professeur Denis Lemieux, S. Lavallée, P. Issalys (dir.), Montréal, Y. Blais, 2018, p. 245-298.

13. R. Dussault, L. Borgeat, Traité de droit administratif, $2^{\mathrm{e}}$ éd., Québec, Presses de l'université Laval, 1984, t. I, partie 2, p. 287. La même terminologie, "acts of administration", a été utilisée dans la version en langue anglaise: R. Dussault, L. Borgeat, Administrative Law: A Treatise, $2^{\mathrm{e}}$ éd., Toronto - Calgary - Vancouver, Carswell, 1985, t. I, partie 2, p. 221.

14. P. Garant, Droit administratif, $6^{\mathrm{e}}$ éd., Montréal, Y. Blais, 2010, p. 159.

15. P. Issalys, D. Lemieux, L'action gouvernementale. Précis de droit des institutions administratives, $3^{\mathrm{e}}$ éd., Montréal, Y. Blais, 2009, p. 143.

16. «La théorie des actes administratifs est donc un effort de systématisation de l'activité juridique de l'administration » (P. Garant, Droit administratif, $7^{\mathrm{e}}$ éd., Montréal, Y. Blais, 2017, p. 160).

17. D. P. Jones, A. S. de Villars, Principles of Administrative Law, $6^{\mathrm{e}}$ éd., Scarborough, Thomson - Carswell, 2014, p. 85.

18. Cour suprême du Canada, Marcotte c. Ville de Longueuil (2009) 3 RCS 65, \$28 (juge LeBel, au nom de la majorité); Cour suprême du Canada, Catalyst Paper Corp. c. District North Cowichan (2012) 1 RCS $5, \$ 12$ (juge en chef McLachlin, au nom de la Cour). Les arrêts de la Cour suprême du Canada sont disponibles en ligne: scc-csc.lexum.com. Les références aux arrêts de la Cour suprême sont composées comme suit: année, volume du recueil dans lequel l'arrêt est publié, abréviation désignant le recueil, puis page dans le recueil. 
prévaloir la dimension organique et formelle afin de présenter le règlement comme un acte juridique d'une entité administrative de type collectivité territoriale. Si la terminologie de l'acte administratif est désormais reconnue en droit canadien, la thématique générale de la motivation des actes administratifs reste largement orientée vers le régime juridique des décisions administratives et des décisions juridictionnelles. Et comme ailleurs, c'est d'abord et avant tout les enjeux liés à la motivation des décisions administratives dites «négatives», assorties de sanctions et de refus, qui retiennent l'attention.

Sur la question de la motivation, l'étude du droit canadien montre clairement la tension entre les deux approches, celle de la solution législative selon l'approche française depuis 1979, par opposition au développement du droit jurisprudentiel où les exigences de motivation sont laissées à l'appréciation du juge judiciaire (le Canada n'offre pas la dualité de juridiction comme en France et dans plusieurs autres systèmes de droit administratif). Cette différence correspond à l'affirmation de deux "modèles forts", le premier étant celui de la prépondérance de la loi en monde romaniste, par opposition à l'explicitation judiciaire des principes du droit des systèmes de droit issus de la filière britannique. Si la France est souvent associée à la première approche, la seconde est surtout représentée par le droit britannique où il n'existe pas, non plus qu'en France ${ }^{19}$, un principe général de motivation ${ }^{20}$. Cette opposition ne repose en définitive que sur le rôle que peut jouer le législateur sans qu'on puisse en déduire une exigence générale de motivation. Même en Grande-Bretagne, le législateur n'est pas resté complètement inactif. Pour la France, l'énumération de la loi du 11 juillet $1979^{21}$ a été conservée dans le Code des relations entre le public et l'administration $^{22}$. La véritable alternative est ailleurs. Le modèle le plus probant et le plus répandu est celui de la codification générale du principe (droit à la motivation pour toutes les décisions défavorables) ${ }^{23}$, comme en témoigne le droit européen ${ }^{24}$, notamment pour l'affirmation du droit à une bonne administration ${ }^{25}$.

Sur ce plan, le Canada offre un portrait plus nuancé, qui témoigne malgré tout de la prépondérance de l'approche jurisprudentielle par fidélité à la tradition de common law. La loi y joue cependant un rôle important et non négligeable. En dépit de nets progrès, la formulation par le législateur d'une exigence générale de motivation n'est pas exempte de difficultés comme le montre la situation qui prévaut au Québec avec la loi sur la justice administrative. Le principe général de motivation obligatoire des décisions administratives défavorables ne vise que «l'Administration gouvernementale» qui est constituée de ministères et organismes dont le gouvernement ou un ministre nomme la majorité des membres et dont le personnel est nommé en vertu de la loi sur la fonction publique ${ }^{26}$. Cette restriction a pour effet d'exclure les collectivités territoriales, les entreprises publiques, les agences, les ordres professionnels, les établissements de santé, ainsi que tout le secteur de l'éducation publique. Dès 1966, l'Alberta avait retenu une approche plus inclusive en prévoyant la motivation pour toute décision défavorable découlant de l'exercice d'un pouvoir conféré par la loi ${ }^{27}$. Ce type de pouvoir fait l'objet de précisions dans les définitions préliminaires. Si la loi est formulée de façon à inclure tout pouvoir administratif ou juridictionnel conféré par une loi, le législateur a néanmoins retenu une liste de six scénarios qui correspondent à des décisions défavorables, ce qui montre, en définitive, l'importance relative de la méthode dite de «liste». L'Ontario et la Colombie-Britannique ont réservé l'exigence de motivation à l'exercice d'une fonction juridictionnelle ${ }^{28}$. Sur ce point, ils rejoignent l'approche britannique qui oblige une série de tribunaux administratifs (énumération en annexe de la loi) à motiver leurs décisions ${ }^{29}$.

En dépit de ces avancées de la loi, plusieurs auteurs canadiens qui analysent les exigences de la motivation en

19. J.-L. Autin, «La motivation des actes administratifs unilatéraux, entre tradition nationale et évolution des droits européens», Revue française d'administration publique, 2011, p. 93 ("L'absence de principe général de motivation»).

20. "There is no general duty to give reasons in English law, although the common law is moving in that direction" (P. Craig, Administrative Law, $8^{\mathrm{e}}$ éd., Londres, Sweet \& Maxwell, 2016, p. 369); voir aussi: P. Cane, Administrative Law, $5^{\mathrm{e}}$ éd., Oxford, Oxford University Press, 2011, p. 88; M. Elliott, «Has the Common Law Duty to Give Reasons Come of Age Yet?», Public Law, 2011, p. 56-74. Si le Tribunals and Inquiries Act, UK, 1992, chap. 53, oblige les ministres à motiver leurs décisions pour le suivi des enquêtes, il n’impose aucune obligation générale de motivation pour les décisions défavorables.

21. Loi $n^{\circ} 79-587$ du 11 juillet 1979 relative à la motivation des actes administratifs...

22. Code des relations entre le public et l'administration, art. L. 211-2 à L. 211-4.

23. E. Untermaier, «Regard critique sur le droit administratif français à l'aune de quelques exemples en droit comparé», in La motivation en droit public, S. Caudal (dir.), Paris, Dalloz, 2013, p. 103.

24. Versions consolidées du traité sur l'Union européenne et du traité sur le fonctionnement de l'Union européenne, Journal officiel de l'Union européenne, C 326/1, 26 octobre 2012, art. 296.

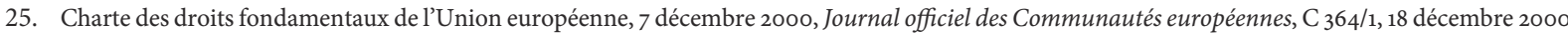
(version remplacée lors de l'entrée en vigueur du traité de Lisbonne, 2010, Journal officiel de l'Union européenne, C 83/389, 30 mars 2010), art. 41.

26. Loi sur la justice administrative, art. 3 et 8 ; loi sur la fonction publique, LRQ, chap. F-3.1.1.

27. "When an authority exercises a statutory power so as to adversely affect the rights of a party, the authority shall furnish to each party a written statement of its decision setting out: a) the findings of fact on which it based its decision, and $b$ ) the reasons for the decisions " (Administrative Procedures and Jurisdiction Act, art. 7).

28. Ontario: loi sur l'exercice des compétences légales, $\$ 17(1)$, qui n'en fait pas une obligation systématique et qui réserve à l'une ou l'autre des parties le droit de demander les motifs; Colombie-Britannique: Administrative Tribunals Act, art. 51 (motivation obligatoire de toutes les décisions).

29. Tribunals and Inquiries Act, art. 10. 
droit administratif orientent souvent leur approche sur le seul droit jurisprudentiel ${ }^{30}$. Malgré le caractère trop restrictif de cette approche, il est néanmoins intéressant de mesurer ce que peut représenter l'évolution du seul droit jurisprudentiel sur la question de la motivation. Si cette analyse peut revêtir un réel intérêt dans la perspective des décisions individuelles, elle ne permet pas de mesurer la portée de l'exigence de la motivation pour les actes de portée générale, notamment pour les actes réglementaires. En dépit de l'absence de toute obligation de motiver pour les fins de l'action réglementaire, une culture de justification a pris le relais pour satisfaire aux exigences de la réglementation intelligente. Dans cette perspective, notre étude permet de comparer les exigences de la motivation avec celles de la justification. Elle montre également la sous-utilisation des lois de portée générale, surtout dans le contexte du droit fédéral.

\section{La prépondérance du contrôle judiciaire pour l'évaluation des exigences de motivation}

Les observateurs sont unanimes afin de reconnaitre à l'arrêt Baker de 1999 le statut de référence obligée dans le domaine de la motivation, et, de façon plus générale, pour les critères relatifs aux exigences de l'équité procédurale ${ }^{31}$. Le cadre juridique de cette affaire relevait du droit de l'immigration. $M^{\text {me }}$ Baker, d'origine jamaïcaine, était entrée au Canada en 1981 à titre de visiteur, mais n'était jamais repartie. Elle avait subvenu illégalement à ses besoins pendant onze années en tant que travailleuse domestique à Toronto, et avait donné naissance à quatre enfants qui étaient tous citoyens canadiens du fait qu'ils étaient nés au Canada. En 1992, suite à la découverte de l'irrégularité de son statut, une ordonnance d'expulsion avait été prise contre elle. En 1993, elle avait demandé d'être dispensée de faire sa demande de résidence permanente de l'extérieur du Canada pour des raisons d'ordre humanitaire (son état de santé et sa situation familiale) sur le fondement d'une disposition de la loi sur l'immigration ${ }^{32}$. Le 18 avril 1994, elle avait reçu une lettre de refus qui concluait qu'il n'y avait pas suffisamment de raisons humanitaires pour traiter sa demande au Canada, sans toutefois donner les motifs de la décision.
Sur la question précise de la motivation, la Cour a rappelé que

[...] la common law a traditionnellement reconnu que l'obligation d'équité n'exige pas, en règle générale, que les décisions administratives soient motivées $[\ldots]^{33}$.

Ce qu'elle avait d'ailleurs constaté dans des arrêts antérieurs. Ces précédents n'avaient pas pour objet des décisions administratives. Dans un arrêt de 1987, le litige avait pour objet une décision du juge en chef du Tribunal du travail qui avait fixé l'audition d'une affaire ailleurs qu'au chef-lieu du district judiciaire où elle avait pris naissance, sans que les parties en soient informées et sans aucun motif ${ }^{34}$. En dépit d'un verdict favorable pour l'appelant, la Cour avait néanmoins rappelé «que, sauf dispositions législatives contraires, les tribunaux judiciaires non plus que les autorités administratives ou quasi judiciaires ne sont tenus de motiver leurs décisions ${ }^{35}$. Cette règle ne s'applique pas lorsque l'obligation de motiver est prévue par la loi, comme ce fut le cas dans l'affaire Northwestern Utilities (1979) ${ }^{36}$ où un tribunal administratif, le Public Utilities Board de l'Alberta, n'avait pas motivé une décision en méconnaissance des exigences de l'Administrative Procedures and Jurisdiction Act. Dans cet arrêt, où l'exigence de motivation découlait de la loi, le juge Estey avait formulé cette remarque, qui a été citée dans Baker:

Cette obligation est salutaire: elle réduit considérablement les risques de décisions arbitraires, raffermit la confiance du public dans le jugement et l'équité des tribunaux administratifs et permet aux parties aux procédures d'évaluer la possibilité d'un appel $[\ldots]^{37}$.

Dans Baker, la décision de rejet était de nature administrative. La motivation des décisions administratives est devenue un enjeu important dans le contexte de l'affirmation des exigences de l'équité procédurale à compter de 1979. Dans le premier arrêt, M. Nicholson, qui occupait un emploi amovible à titre d'agent de police, avait été congédié sans avis préalable, sans motif et sans audition. Les juges majoritaires ont affirmé pour la première fois le droit à l'équité procédurale en matière administrative, notamment pour la violation de la règle Audi alteram partem, mais également pour l'absence de motifs ${ }^{38}$. Dans une autre affaire relevant du droit carcéral, deux détenus ayant participé à une prise d'otages avaient été transférés

30. Ce sont majoritairement des contributions anglo-canadiennes: D. P. Jones, A. S. de Villars, Principles of Administrative Law, p. 348-363 (les deux auteurs évoquent néanmoins les exigences de la loi à la lumière du droit de l'Alberta, p. 354); K. Lo, «When Efficiency Calls: Rethinking the Obligation to Provide Reasons for Administrative Decisions», Queen's Law Journal, vol. 43, n 2, 2018, p. 325-367; M. Liston, «"Alert, Alive and Sensitive": Baker, the Duty to Give Reasons, and the Ethos of Justification in Canadian Public Law ", in The Unity of Public Law, D. Dyzenhaus (dir.), Portland, Hart, 2004, p. 113-141.

31. Cour suprême du Canada, Baker c. ministre de la Citoyenneté et de l'Immigration du Canada (1999) 2 RCS 817 (juge L'Heureux-Dubé au nom de la majorité, le juge Iacobbucci étant dissident sur l'application de la Convention relative aux droits de l'enfant en droit interne).

32. Loi sur l'immigration, LRC 1985, chap. I-2, $\$ 114(2)$.

33. Cour suprême du Canada, Baker..., $\$ 37$.

34. Cette décision a été invalidée pour violation de la règle Audi alteram partem.

35. Cour suprême du Canada, Les supermarchés Jean Labrecque Inc. c. le Tribunal du travail et le juge Bernard Lesage (1987) 2 RCS 219 , $\$ 46$ (juge L'Heureux-Dubé au nom de la Cour). La Cour a évalué que la fixation de la date et du lieu du procès n'est pas un acte de pure administration.

36. Cour suprême du Canada, Northwestern Utilities Ltd. c. Ville d'Edmonton (1979) 1 RCS 684 (juge Estey).

37. Ibid., p. 706; Cour suprême du Canada, Baker..., $\$ 38$.

38. Cour suprême du Canada, Nicholson c. Haldiman-Norfolk Regional Board of Commissioners of Police (1979) 1 RCS 311 (juge Laskin au nom de la majorité). 
dans un établissement à sécurité maximale et placés en isolement dans l'unité spéciale de détention (USD). En dépit d'une recommandation favorable du Conseil des cas de ségrégation administrative afin de réintégrer ces deux détenus dans la population carcérale, le directeur de l'établissement Kent les avait maintenus en USD sans leur donner aucun motif, ni la possibilité d'être entendus ${ }^{39}$. À l'unanimité, la Cour a sanctionné ce refus pour violation des exigences de l'équité procédurale, notamment à cause de l'absence d'audition. Sans que la Cour prenne position sur une exigence générale de motivation, elle associait déjà le droit d'obtenir des motifs au respect de l'équité procédurale. Au nom de la Cour, le juge Le Dain avait affirmé en fin d'arrêt que le droit à une audition équitable constituait «un droit distinct et absolu ${ }^{40}$, ce qui laissait à tout le moins supposer que le caractère équitable devait résulter de la divulgation des motifs afin de permettre un échange entre les parties. Mais la Cour n'avait pas formulé une position explicite sur cette question.

Dans la perspective de cet échange d'information entre les parties, l'arrêt Knight (1990) constitue une étape plus significative ${ }^{41}$. M. Knight, en sa qualité de directeur de l'enseignement, avait été démis de ses fonctions sur préavis de trois mois. En alléguant un manquement à l'équité procédurale, il avait poursuivi le Conseil (Board of Education) pour congédiement injustifié et illégal. Au nom des juges formant la majorité, la juge L'Heureux-Dubé a constaté que ce droit à l'équité procédurale ne pouvait pas résulter de la loi ${ }^{42}$ ou du contrat d'emploi, mais de facteurs généraux liés au fait que l'employeur est un organisme public qui doit exercer ses pouvoirs en conformité avec les exigences du droit administratif. En tenant compte de ces facteurs ${ }^{43}$, elle est parvenue à la conclusion que le Conseil avait le devoir général d'agir équitablement, ce qui justifiait la nécessité de donner les motifs du renvoi. Malgré cette position de principe, elle a retenu une solution pragmatique puisque, dans les circonstances propres à cette affaire, le Conseil n'était pas soumis à l'exigence de donner « un avis en bonne et due forme de ses motifs » puisque les parties avaient été en mesure de se rencontrer dans le but de négocier un nouveau contrat ${ }^{44}$. Dans ce contexte, il n'était pas nécessaire de parvenir à l'exhaustivité dans la formulation des motifs ${ }^{45}$.

En dépit du fait que l'arrêt Knight a été explicitement renversé par la Cour en 2008 afin de soustraire le champ contractuel de l'application des règles de l'équité procédurale $^{46}$, il a permis de préciser des balises en matière de motivation. Dans l'arrêt Baker, la situation factuelle était, à toutes fins utiles, identique, puisque le refus de l'agent d'immigration n'était pas motivé. Mais, comme elle l'avait fait dans l'arrêt Knight, la Cour a évité le formalisme en constatant que «l'obligation de donner des motifs a été remplie [...] par la production des notes de l'agent Lorentz à l'appelante» ( $\mathrm{M}^{\mathrm{me}}$ Baker), suite aux démarches de son avocat ${ }^{47}$. L'obtention de ces notes a été déterminante pour l'issue du litige puisqu'elle a permis d'établir la partialité du décideur.

Sur le plan des principes, l'arrêt Baker reste la référence obligée pour les conditions relatives à l'équité procédurale $^{48}$, ainsi que pour le droit à la motivation, puisque la Cour a adopté une approche plus favorable en affirmant qu'

[...] il est maintenant approprié de reconnaître que, dans certaines circonstances, l'obligation d'équité procédurale requerra une explication écrite de la décision ${ }^{49}$.

Il ne s'agit pas d'une obligation générale afin de motiver toute décision défavorable. Pour justifier l'exigence de la motivation, il faut tenir compte de l'importance de la décision pour l'intéressé, ainsi que l'existence d'un droit d'appel prévu par la loi, ou d'autres éléments liés aux circonstances. Pour les éléments de contexte, la position de la Cour est un peu approximative, bien que, sur ce point, il est plus facile de montrer que "l'importance cruciale d'une décision d'ordre humanitaire», comme c'était le cas pour $\mathrm{M}^{\mathrm{me}}$ Baker, «milite en faveur de l'obligation de donner des motifs $»^{50}$. La Cour a également pris soin de préciser que la «transparence peut être atteinte de différentes façons $[. .$.$] en tenant compte de la réalité quotidienne des$ organismes administratifs $»^{51}$. Cette nuance est essentielle puisque, dans les conditions requises pour le respect des

39. Cour suprême du Canada, Cardinal et Oswald c. Directeur de l'établissement Kent (1985) 2 RCS 643.

40. «Il faut considérer le droit à une audition équitable comme un droit distinct et absolu qui trouve sa justification essentielle dans le sens de la justice en matière de procédure à laquelle toute personne touchée par une décision administrative a droit » (Cour suprême du Canada, Cardinal et Oswald..., p. 661)

41. Cour suprême du Canada, The Board of Education of the Indian Head School Division n ${ }^{\circ} 19$ of Saskatchewan c. Knight (1990) 1 RCS 653 (ci-après Knight).

42. The Education Act, RSS, 1978, chap. E-o.1.

43. La nature de la décision, la relation entre l'organisme et l'individu, l'effet de la décision pour les droits de l'individu (la Cour, prudente, n'a pas utilisé le terme «citoyen») (Cour suprême du Canada, Knight, p. 669).

44. Cour suprême du Canada, Knight, p. 686 .

45. «[...] l'obligation de donner des motifs ne comporte pas nécessairement la révélation complète par l'organisme administratif de toutes les raisons du renvoi de l'employé; il s'agit plutôt de lui communiquer les raisons générales de manière à lui indiquer en substance ce qui a motivé le renvoi» (ibid., p. 685).

46. Cour suprême du Canada, Dunsmuir c. Nouveau-Brunswick (2008) 1 RCS 190, $\$ 81$ (juges Bastarache et Lebel).

47. Cour suprême du Canada, Baker..., $\$ 44$.

48. La Cour a énuméré cinq conditions : 1) La nature de la décision recherchée et le processus suivi; 2) La nature du régime législatif; 3) L’importance de la décision pour les personnes visées; 4) Les attentes légitimes de la personne qui conteste la décision; 5) L'analyse des procédures requises. La Cour indique que cette liste n'est pas exhaustive.

49. Cour suprême du Canada, Baker..., $\$ 43$.

50. Ibid.

51. Ibid., $\$ 44$. 
exigences de l'équité procédurale, la Cour précise qu' «[i]l n'est plus nécessaire, sauf peut-être lorsque la loi l'exige, de faire la distinction entre les décisions judiciaires, quasi judiciaires et administratives ${ }^{52}$. Si cette position offre les avantages de la simplicité, il faut tenir compte, pour les exigences de la motivation, de l'ouverture manifestée par la Cour à l'égard de la diversité des organismes administratifs et des procédures administratives à des fins de «souplesse» ${ }^{53}$. En explicitant en 2008 les conditions requises pour l'application de la règle de la déférence, la Cour a rappelé que celle-ci «suppose plutôt le respect du processus décisionnel au regard des faits et du droit ${ }^{54}$.

Dans les années postérieures, la Cour a invalidé deux décisions administratives qui n'étaient pas motivées. En 2002, dans Suresh, elle a jugé insuffisante une décision d'expulsion fondée sur le fait que l'appelant, en raison de son appartenance aux Tigres tamoul, représentait «un danger pour la sécurité du Canada» en lien avec le «terrorisme», avec pour seul justificatif une note de service produite par un agent d'immigration. Compte tenu du fait que Suresh était susceptible d'être expulsé vers un pays où il risquait d'être torturé, il pouvait à juste titre invoquer les garanties de l'article 7 (droit à la vie, à la liberté et à la sécurité) de la Charte canadienne des droits et libertés en matière de justice fondamentale. Dans cet arrêt unanime, la Cour conclut que «la ministre doit motiver sa décision par écrit $»^{55}$. Elle précise que les

[...] motifs doivent exposer clairement et étayer rationnellement sa conclusion qu'il n'existe pas de motifs sérieux de croire que la personne [...] sera torturée ou exécutée ou subira quelque autre traitement cruel ou inusité [...]

dans la mesure où l'intéressé exprime cette crainte. Elle ajoute que

[...] les motifs doivent également préciser les raisons pour lesquelles la ministre croit que l'intéressé constitue un danger pour la sécurité du Canada comme l'exige la loi ${ }^{56}$.

Puisque cette décision portait atteinte à des droits garantis par l'article 7 , des garanties procédurales plus strictes devaient être observées.

Dans Lafontaine (2004), une atteinte à un droit reconnu par la Charte avait également été alléguée (la liberté de religion protégée par l'alinéa 2a), puisque la municipalité avait refusé de modifier le règlement de zonage afin d'accommoder la congrégation des Témoins de Jéhovah pour l'édification d'un lieu de culte, faute de terrain disponible dans la zone communautaire régionale. En alléguant qu'elle était dans l'impossibilité de trouver un terrain dans cette zone, la congrégation avait demandé à plusieurs reprises la modification du règlement de zonage. La municipalité avait répondu par la négative en affirmant qu'elle n'avait aucune obligation de motiver sa décision. Selon les juges majoritaires, le devoir d'équité procédurale obligeait la municipalité à motiver ses refus ${ }^{57}$. Au nom de la majorité, la juge en chef McLachlin affirme que

La communication des motifs [...] favorise la transparence et l'équité du processus décisionnel, réduit les risques de décisions arbitraires ou abusives, et entretient la confiance qu'éprouvent les citoyens envers les agents de l'État ${ }^{58}$.

Sur le plan de l'équité procédurale et de la motivation, ces deux arrêts montrent déjà des différences significatives puisque les exigences de la justice fondamentale, appliquées dans le premier, ne pouvaient pas être invoquées dans le second. Les arrêts postérieurs à Baker témoignent de cette modulation qui caractérise la démarche du juge dans les systèmes de droit issus de la filière britannique. Malgré le fait que la Cour ne tienne plus compte de la distinction entre fonction administrative et fonction juridictionnelle afin de déterminer si les règles de l'équité procédurale doivent être appliquées, elle adopte, pour les fins de la motivation, une autre approche, très pragmatique, centrée directement sur l'analyse du type de procédure. Dans l'exercice d'une fonction juridictionnelle, comme c'est le cas par exemple pour les tribunaux administratifs et les arbitres où les décisions sont motivées, les attentes ne seront pas du même type, car la Cour peut se montrer plus tolérante, même si la motivation n'est pas exhaustive. L'arrêt Newfoundland de 2011 en est un exemple. Il avait pour objet la contestation par un syndicat de la décision rendue par un arbitre à propos des motifs, jugés insuffisants par la partie syndicale, relatifs au calcul du nombre de congés annuels payés. En reprenant la grille d'analyse développée dans Dunsmuir (2008) à propos du caractère raisonnable de la décision (cet élément conditionne la déférence judiciaire), la Cour précise que la position retenue en 2008 « témoigne d'une reconnaissance respectueuse du vaste éventail de décideurs spécialisés qui rendent couramment des décisions ${ }^{59}$. La Cour en conclut que l'insuffisance des motifs ne permet pas «à elle seule de casser une décision ${ }^{60}$. Afin de satisfaire à l'exigence de la

52. Cour suprême du Canada, Knight, p. 669.

53. Cour suprême du Canada, Baker..., $\$ 44$.

54. Cour suprême du Canada, Dunsmuir..., $\$ 47$.

55. Cour suprême du Canada, Suresh c. ministre de la Citoyenneté et de l'Immigration du Canada (2002) 1 RCS $3, \$_{126 .}$

56. Ibid.

57. Cour suprême du Canada, Congrégation des témoins de Jéhovah de St-Jérôme-Lafontaine c. Village de Lafontaine (2004) 2 RCS 650, $\$ 12$.

58. Ibid., $\$ 13$. Cette formule est proche de celle utilisée par le juge Estey en 1979 dans l'affaire Northwestern Utilities. L'absence de motifs ne constitue pas forcément une issue déterminante dans quelques litiges. Dans l'arrêt Centre hospitalier Mont-Sinaï c. ministre de la Santé et des Services sociaux, 2001, 2 RSC 281, la modification du permis n'avait fait l’objet d'aucun motif ( $\$ 21$, juge Binnie). Les juges formant la majorité ont suivi un autre raisonnement en affirmant que le ministre était lié par sa décision initiale.

59. Cour suprême du Canada, Newfoundland and Labrador Nurses' Union c. Conseil du Trésor de Terre-Neuve-et-Labrador (2011) 3 RCS 708, $\$ 13$ (juge Abella au nom de la Cour).

60. Ibid., $\$ 14$. 
motivation, elle précise que l'exhaustivité n'est pas requise dans l'évaluation de tous les arguments avancés par les parties, ainsi que pour le raisonnement suivi par le décideur ${ }^{61}$. Dans cette affaire, la question de l'équité procédurale a été soulevée uniquement en Cour suprême. En reprenant les critères élaborés dans Baker, la Cour précise qu'elle n'a "pas affirmé que des motifs s'imposent dans tous les cas, ni que leur qualité relève de l'équité procédurale ${ }^{62}$. Dans cette perspective, sa démarche montre une volonté de modulation des exigences de la motivation.

À la lumière de cette approche respectueuse des particularismes de chaque organisme et du type de décision, la Cour a répondu par la négative à des contestations fondées sur l'absence de motifs, ou encore à des allégations relatives à leur insuffisance. Dans l'arrêt Mavi, également rendu en 2011, la Cour a jugé que la motivation n’était pas nécessaire pour une décision administrative relative au recouvrement d'une créance suite au non-respect des conditions de plusieurs demandes de parrainage en matière d'immigration ${ }^{63}$. Dans un tout autre contexte procédural, le commissaire à l'information et à la protection de la vie privée de l'Alberta avait prorogé de façon explicite la durée d'une enquête bien après le délai de quatre-vingt-dix jours requis par la loi ${ }^{64}$. Cette enquête avait pour objet des plaintes dirigées contre l'Alberta Teachers Association. La prorogation après l'expiration du délai était implicite et n'était pas motivée. La Cour a néanmoins tenu compte des décisions déjà rendues, à maintes reprises, par le commissaire et ses délégués sur cette question, afin d'examiner le fondement raisonnable de la décision de prorogation, qu'elle a par ailleurs validée ${ }^{65}$. Cette reconstruction a posteriori de la motivation requise, quoique critiquée ${ }^{66}$, montre que la démarche suivie en matière de contrôle judiciaire doit être caractérisée par la souplesse et non par le formalisme.

Ce pragmatisme caractérise également les derniers arrêts. Dans Agraira (2013), le ministre de la Sécurité publique et de la Protection civile avait rejeté une demande de dispense formulée par M. Agraira, un citoyen de la Lybie, suite à l'interdiction de territoire prononcée contre lui pour cause d'appartenance à une organisation terroriste. Dans cet arrêt unanime, la Cour a estimé qu'en dépit de leur brièveté, «les motifs du ministre étaient justifiables, transparents et intelligibles », car l'interprétation implicite de l'intérêt national reposait en définitive sur des considérations de sécurité nationale et de sécurité publique, ainsi que sur d'autres éléments énoncés dans un guide opérationnel relatif à l'examen des demandes de dispense ministérielle ${ }^{67}$. Dans McLean rendu la même année, la Commission des valeurs mobilières de Colombie-Britannique n'avait pas motivé une ordonnance de réciprocité (2010) dirigée contre $\mathrm{M}^{\mathrm{me}} \mathrm{McLean}$, suite à une première ordonnance rendue en 2005 par la Commission des valeurs mobilières de l'Ontario pour cause d'inconduite dans l'exercice de ses fonctions de dirigeante au sein d'une entreprise ontarienne ${ }^{68}$. Comme il s'agissait de deux ordonnances jumelles, $\mathrm{M}^{\mathrm{me}}$ McLean était interdite d'accomplir les mêmes actes dans les deux provinces pour la même période. Comme l'instance principale avait été initiée en Ontario, celle engagée en Colombie-Britannique a été décrite comme une instance secondaire dans le cadre d'un régime de coopération interprovinciale. Ce contexte un peu particulier explique que la cour n'ait pas jugé utile de censurer l'absence de motifs, notamment parce qu' «il n'y a aucun avantage à renvoyer l'affaire [à la Commission] pour qu'elle explique ce qui est déjà manifeste ${ }^{6}{ }^{9}$. Ces deux décisions résultaient de l'exercice d'une fonction juridictionnelle car il s'agit de tribunaux administratifs.

Enfin, l'arrêt Capilano, rendu en 2016, met également en lumière une autre décision rendue sans motifs dans l'exercice d'une fonction juridictionnelle ${ }^{70}$. Un centre commercial situé à Edmonton (Alberta) avait contesté la valeur imposable fixée par la ville en déposant une plainte auprès du Comité de révision des évaluations. En alléguant des erreurs dans ce processus d'évaluation, la ville d'Edmonton avait demandé une révision à la hausse de la valeur imposable. Le Comité de révision avait acquiescé en partie à cette demande sans justifier qu'il avait le pouvoir d'augmenter l'évaluation. Sur le plan des principes, les juges formant la majorité ont rappelé que «lorsqu'un tribunal ne motive pas sa décision, il devient plus difficile de se prononcer sur sa justification et sur son intelligibilité ${ }^{71}$, tout en ajoutant, par un renvoi à l'arrêt Newfoundland ${ }^{72}$, que "l'absence complète de motifs équivau[ $\mathrm{t}$ ] à une erreur de droit » lorsque cette motivation est requise par les exigences de l'équité procédurale ${ }^{73}$. Mais puisque l'avocat représentant le centre commercial

61. Ibid., $\$ 16-17$.

62. Ibid., $\$ 20$.

63. «Vu le cadre législatif et réglementaire, la nature non judiciaire du processus et l'absence de tout droit d'appel légal, l'obligation d'équité faite à l'État dans le cas qui nous occupe ne va pas jusqu'à le contraindre à motiver chacune de ses décisions» (Cour suprême du Canada, Procureur général du Canada c. Mavi (2011) 2 RCS 504, \$45).

64. Cour suprême du Canada, Information and Privacy Commissioner of Alberta c. Alberta Teacher's Association (2011) 3 RCS 654.

65. «En l'occurrence, l'examen des motifs du commissaire ou de ses délégués dans d'autres affaires permet de déterminer sans peine que la décision implicite de la déléguée [du commissaire] a une assise raisonnable» (Cour suprême du Canada, Information and Privacy Commissioner of Alberta..., \$56).

66. K. Lo, «When Efficiency Calls...», p. 340 et 354

67. Cour suprême du Canada, Agraira c. ministre de la Sécurité publique et de la Protection civile (2013) 2 RCS 559, $\$ 60$ et 89 .

68. Cour suprême du Canada, McLean c. British Columbia Securities Commission (2013) 3 RCS 895.

69. Ibid., $\$ 72$.

70. Cour suprême du Canada, Ville d'Edmonton c. Edmonton East (Capilano) Shopping Centres Ltd. (2016) 2 RCS 293 (ci-après Capilano).

71. Ibid., $\$ 36$.

72. Cour suprême du Canada, Newfoundland..., \$20-22.

73. Cour suprême du Canada, Capilano, $\$ 37$ (juge Karakatsanis au nom de la majorité). 
Capilano avait reconnu au Comité le pouvoir de réduire ou d'augmenter les évaluations, la Cour a estimé que cette question ne pouvait plus être examinée sur ce plan (motivation explicite), mais sur le fondement de son caractère raisonnable à la lumière des pouvoirs accordés par la loi au Comité.

Cet arrêt, comme les précédents, montre les impératifs propres au contrôle judiciaire lorsqu'il s'agit de déterminer la norme de la décision correcte ou de la décision raisonnable suivant la grille retenue dans l'arrêt Dunsmuir. Ces impératifs requièrent un examen a posteriori de la décision rendue à la lumière de tous les éléments qui conditionnent sa régularité et sa validité. La rationalité qui préside à cet examen repose sur l'analyse contextuelle. La Cour suprême est inévitablement entraînée dans ce qui peut paraître comme une démarche casuistique, puisqu'elle est saisie au cas par cas de demandes de contrôle judiciaire où la question des motifs n'est pas forcément un élément central du litige. Les différentes cours d'appel (Cour d'appel fédérale, cours d'appel des provinces) doivent procéder à ce type d'examen en fonction de nombreux critères (nature des dispositions habilitantes, objectifs de la loi, caractéristiques du processus décisionnel, expertise du décideur, équité procédurale, pour donner quelques exemples).

Cette analyse contextuelle répond avant tout aux exigences de la primauté du droit en tenant compte des règles du droit administratif, et parfois également, des règles issues du contentieux constitutionnel, si la justice fondamentale peut être invoquée. Par ses finalités qui sont légitimes et nécessaires, cette approche est très différente de la démarche qui résulte de l'utilisation de la loi afin d'obliger, a priori, les autorités administratives à motiver leurs décisions. Cette autre approche est directement centrée sur les citoyens afin de faire prévaloir le principe de sécurité juridique et l'intelligibilité du droit. En dépit de ses limites, la loi sur la justice administrative (art. 8) en est un exemple en obligeant les autorités administratives à motiver toutes les décisions défavorables dans l'exercice d'une fonction administrative.

Cette analyse comparative peut sembler avoir été négligée dans des études récentes. Une remontée dans le temps montre néanmoins qu'il n'en a pas toujours été ainsi. Dans une étude qui constitue une référence dans ce domaine, le professeur Kushner avait comparé l'approche législative (statutory requirements) avec la position traditionnelle de la common law (no general obligation to give reasons for a decision). Déjà, les questions essentielles avaient été soulevées en visant explicitement le rôle du juge:
Upon which decision makers should the obligation be imposed? Should there be exceptions? What are adequate reasons? What is the effect of failure to comply ${ }^{74}$ ?

Il avait souligné que ces interrogations, qui conservent toute leur acuité, ne pouvaient être résolues dans l'abstrait: "Again the courts may be reluctant to answer these questions without at least some guidance from the legislatures " 75 . Dans une étude antérieure faite au Québec, la législation canadienne avait été comparée avec celle de Grande-Bretagne et des États-Unis, afin de suggérer la motivation obligatoire de toutes les décisions administratives par le recours à la $\operatorname{loi}^{76}$.

Dans leurs commentaires, les observateurs privilégient le rôle déterminant du juge, tout en rappelant, le cas échéant, les interventions du législateur. Depuis l'affaire Baker, la flexibilité retient l'attention ${ }^{77}$. Il n'y a pas vraiment d'analyse comparative des avantages réciproques de la loi et de la jurisprudence fondée sur une étude plus exhaustive du droit comparé. En 1998, les professeurs Macdonald et Lametti avaient néanmoins insisté sur la nécessité de distinguer les décisions administratives des décisions juridictionnelles afin de ne pas uniformiser les exigences de motivation sans égard à la diversité des organismes publics ${ }^{78}$. Afin de résoudre ces difficultés, ils ont préconisé une approche contextuelle fondée sur la spécificité de chaque processus décisionnel au détriment d'une approche plus générale. Si cette démarche correspond aux exigences du contrôle judiciaire, elle est en revanche très éloignée des avantages que représente une intervention législative afin de formuler des règles simples et claires pour les citoyens. Au Québec, l'exigence de motivation prévue par la loi sur la justice administrative a été décrite comme " une avancée notable $»^{79}$. Le recours à une formulation simple et générale offre un net contraste avec la culture casuistique héritée de la common law.

\section{Les exigences de justification en matière réglementaire}

Dans sa première série d'arrêts relatifs à l'équité procédurale, la Cour suprême a exclu les actes de portée générale du champ de la motivation. Dans l'affaire Inuit Tapirisat (1980), elle était parvenue à la conclusion que les règles de l'équité procédurale ne pouvaient être invoquées dans le domaine de l'élaboration des politiques publiques ${ }^{80}$. Le Cabinet n'est pas tenu de les respecter pour évaluer la pertinence d'un appel dirigé contre une décision du

74. H. L. Kushner, «The Right to Reasons in Administrative Law», Alberta Law Review, vol. 24, n 2, 1986, p. 332.

75. Ibid.

76. M.-C. Lévesque-Crevier, «La motivation en droit administratif», Revue du Barreau, n 40, 1980, p. 621.

77. P. Bryden, «Standards of Review and Sufficiency of Reasons: Some Practical Considerations », Canadian Journal of Administrative Law and Practice, $\mathrm{n}^{\circ}$ 19, 2006, p. 191

78. R. A. Macdonald, D. Lametti, «Reasons for Decision in Administrative Law», Canadian Journal of Administrative Law and Practice, ${ }^{\circ}$ 3, 199o, p. 123 sq.

79. P. Issalys, D. Lemieux, L'action gouvernementale..., p. 956.

80. Cour suprême du Canada, Procureur général du Canada c. Inuit Tapirisat of Canada (1980) 2 RCS $735-758$ (juge Estey au nom de la Cour) 
Conseil de la radiodiffusion et des télécommunications du Canada (CRTC) qui a pour objet une réglementation de nature tarifaire ${ }^{81}$. La Cour a été plus explicite dans Knight en affirmant que, pour le devoir d'agir équitablement,

Les décisions d'une nature législative et générale peuvent à cet égard être distinguées des actes de nature plus administrative et particulière, qui ne comportent pas une telle obligation $^{82}$.

Si les exigences de l'équité procédurale, ainsi que les garanties offertes par la motivation de différentes catégories de décisions administratives et juridictionnelles, ne s'appliquent pas pour l'élaboration des actes de portée générale comme les règlements, la question de la motivation ne revêt plus le même sens à la lumière de cette culture de justification qui caractérise l'évolution du droit public contemporain. Le phénomène de la réglementation intelligente repose sur une approche de justification aux fins de l'élaboration de nouveaux règlements ${ }^{83}$. À bien des égards, la justification est plus exigeante que la motivation puisqu'il faut démontrer la pertinence économique et scientifique d'un règlement, alors qu'en contrepartie, la motivation requiert des références précises aux éléments de fait et de droit qui conditionnent la légalité d'une décision. La justification se situe plus en amont afin de montrer la nécessité d'un dispositif normatif de type réglementaire. Afin de maximiser les avantages escomptés, l'administration fédérale doit recenser et évaluer les incidences économiques, environnementales et sociales de la réglementation.

Dans la «Directive du Cabinet sur la réglementation» (2018) dont l'application relève du Secrétariat du Conseil du Trésor, les ministères et organismes fédéraux doivent démontrer que «l'intervention du gouvernement est nécessaire» "en s'appuyant sur les meilleures données et analyses disponibles ${ }^{84}$. Au niveau des principes directeurs, les ministères et organismes doivent utiliser des «données probantes» afin que les propositions formulées en matière réglementaire puissent être «fondées sur des éléments de preuve, une analyse rigoureuse des coûts et des avantages ", ainsi qu' " une évaluation des risques » ${ }^{85}$. Cette exigence de l'évaluation préalable repose sur l'élaboration de l'étude d'impact de la réglementation (EIR) ${ }^{86}$.

\section{L'EIR a pour objet l'évaluation}

[...] des répercussions favorables ou néfastes possibles d'un projet de règlement sur la santé, la sûreté et la sécurité, le bien-être socioéconomique des Canadiens et des entreprises ainsi que sur l'environnement.

Elle doit également offrir « une analyse qualitative et quantitative proportionnelle aux répercussions prévues $\mathrm{du}$ projet de règlement ${ }^{87}$. Enfin, une analyse coûts / bénéfices est nécessaire. Si la «Politique sur l'élaboration de la réglementation» (2018) est un peu plus exhaustive ${ }^{88}$, c'est en réalité la «Politique sur l'analyse coûts-avantages» $(2018)^{89}$ qui permet de mesurer la volonté des autorités fédérales de parvenir à une analyse "proportionnelle» fondée sur « une évaluation qualitative des coûts et des avantages, qui peuvent être quantifiés ou convertis en valeur monétaire, si les données sont disponibles » ${ }^{\circ}$. Dans le «Guide d'analyse coûts-avantages pour le Canada $»^{91}$ qui remonte à 2007 , la méthode privilégiée est celle du "principe de la volonté de payer» (VDP). Cette évaluation est étroitement liée au concept de "rente du consommateur». Cette rente résulte de la différence entre ce que les consommateurs sont disposés à payer pour obtenir un bien ou un service et le prix qu'ils payent réellement sur le marché. Si le VDP constitue le principal outil d'évaluation, le «Guide» reconnaît néanmoins que l'évaluation est plus difficile pour des interventions de nature réglementaire dans les domaines de l'environnement, de la santé et de la sécurité en raison de l'absence de marché. C'est dans ce contexte que d'autres méthodes sont suggérées, notamment la méthode des prix hédonistes, la méthode du coût de trajet, la méthode du comportement d'évitement, ainsi que la méthode du coût de la maladie. Ces méthodes sont avant tout de nature quantitative. Sur ce plan, le «Guide» reconnaît que certains avantages peuvent se révéler trop difficiles à quantifier en termes monétaires. Il peut exister des variables incertaines sur les résultats d'une intervention réglementaire (risques potentiels et incertitudes scientifiques).

Dans une perspective chronologique, le Canada, à titre d'État membre de l'Organisation de coopération et de développement économiques (OCDE), a été l'un des premiers à systématiser le recours à l'étude d'impact avant

81. Le paragraphe 64(1) de la loi nationale sur les transports, LRC 1970, chap. N-17, offrait cette possibilité qui a été conservée en partie par le $\$ 28(1)$ de la loi sur la radiodiffusion, LC 1991, chap. 11, puisque le Cabinet peut, de sa propre initiative, modifier une décision du CRTC.

82. Cour suprême du Canada, Knight, p. 670.

83. D. Mockle, «La réglementation intelligente : réglementer mieux ou réglementer moins? », Revue française de droit administratif, 2015 , p. 1225 sq.

84. Secrétariat du Conseil du Trésor du Canada, «Directive du Cabinet sur la réglementation », Ottawa, 2018, art. 5.1.1, en ligne: https://www.canada.ca/ $\mathrm{fr} /$ secretariat-conseil-tresor/services/gestion-reglementation-federale/lignes-directrices-outils/directive-cabinet-reglementation.html.

85. Ibid., art. 3 .

86. Ibid., art. 5.2

87. Ibid.

88. Secrétariat du Conseil du Trésor du Canada, «Politique sur l'élaboration de la réglementation», Ottawa, 2018, en ligne: https://www.canada.ca/ $\mathrm{fr} /$ secretariat-conseil-tresor/services/gestion-reglementation-federale/lignes-directrices-outils/politique-elaboration-reglementation.html.

89. Secrétariat du Conseil du Trésor du Canada, «Politique sur l'analyse coûts-avantages», Ottawa, 2018, en ligne: https://www.canada.ca/fr/ secretariat-conseil-tresor/services/gestion-reglementation-federale/lignes-directrices-outils/politique-analyse-couts-avantages.html.

90. Ibid., art. 6.1.2.

91. Secrétariat du Conseil du Trésor du canada, «Guide d'analyse coûts-avantages pour le Canada: propositions de réglementation », Ottawa, 2007, en ligne: https://www.tbs-sct.gc.ca/rtrap-parfa/analys/analys-fra.pdf. 
l'apparition de la «Directive du Cabinet sur la rationalisation de la réglementation» de $2007^{92}$. Le recours à cette technique (regulatory impact assesment) remonte à 1978 avec une première directive du Cabinet qui constituait un projet-pilote ${ }^{93}$, suivie en 1986 par la première mouture de la politique de réglementation qui résultait d'une initiative du secrétariat du Conseil du Trésor ${ }^{94}$. À la fin de la décennie 1990, l'expérience canadienne était considérable, tout en restant cantonnée aux énoncés de politiques du Conseil du Trésor. Le manque d'intérêt pour un cadre législatif est manifeste. La loi sur les textes réglementaires n'a pas été modifiée ${ }^{95}$. Dans le défunt projet de loi sur les règlements déposé en 1995, les autorités fédérales n'avaient pas jugé utile d'insérer des dispositions spécifiques sur les études d'impact de la réglementation ${ }^{96}$.

Trop souvent confinée à une rationalisation économique et chiffrée de la réglementation, l'étude d'impact n'engendre pas les résultats escomptés. L'adhésion sans nuance à des méthodes de calcul fondées exclusivement sur des valeurs quantifiables est critiquée ${ }^{97}$. Comme le préconise l'OCDE dans ses publications de la dernière décennie, la réglementation intelligente doit prendre en compte les difficultés liées à l'évaluation des impacts sociaux et environnementaux. Dans la perspective de la biodiversité, l'OCDE constate que la valeur des biens publics est souvent sous-estimée du fait que les impacts non marchands sont monétisés en fonction de la somme des préférences individuelles ${ }^{98}$. Le Canada a tenu compte de ces enjeux en intégrant l'évaluation de dimensions non monétaires, comme les répercussions sur l'environnement, ainsi que les répercussions socioéconomiques selon les identités de genre (analyse comparative entre les sexes), dans la dernière mouture de la politique de réglementation de $2018^{99}$.

Malgré le fait que la justification de l'action réglementaire repose sur d'autres prémisses que la motivation des actes individuels, il est possible d'arguer qu'il n'y a pas de différence fondamentale entre la motivation et la justification puisqu'elles obéissent à une rationalité qui requiert de résoudre la question de l'opportunité par divers types de motifs. Ce ne serait donc qu'une question d'échelle dans la recherche des motifs, notamment dans l'évaluation du contexte factuel. À titre d'exemple, une décision administrative dite "individuelle» peut faire appel à plusieurs considérations techniques et scientifiques afin d'attribuer ou retirer un permis. Dans le domaine de la santé et de la salubrité, ces considérations sont déterminantes.

Le niveau de justification requise pour les actes réglementaires va néanmoins plus loin puisqu'il faut

[...] démontrer que la mesure réglementaire constitue le meilleur outil pour réaliser les objectifs de politique publique souhaités avec l'appui de données et d'analyses ${ }^{100}$.

L'analyse coûts / avantages inclut «le choix de l'instrument», notamment dans le but «d'évaluer l'efficacité et la justesse des instruments réglementaires et non réglementaires qui permettront d'atteindre des objectifs stratégiques ${ }^{101}$. Dans la " Politique sur l'élaboration de la réglementation », «les organismes de réglementation doivent considérer des solutions de rechange au règlement», notamment par la sélection des instruments appropriés qui ne sont pas forcément des mesures législatives et / ou réglementaires, mais, le cas échéant, des «mécanismes volontaires, normes, lignes directrices, directives et politiques ${ }^{102}$. Cette approche est centrée sur l'efficacité technique de chaque instrument de rechange à la réglementation. Le type de justification requis n'a plus rien à voir avec les enjeux traditionnels de la motivation des actes individuels puisque c'est la pertinence de l'action réglementaire qui est en cause. Lorsque les autorités administratives doivent rendre des décisions administratives ou juridictionnelles, elles ne sont pas tenues de trouver des solutions de rechange. Elles ont l'obligation de rendre une décision.

La recherche de ces solutions de rechange n'est pas exempte de présupposés hostiles à la réglementation. L'éclectisme dans le choix des moyens d'intervention reflète la logique des Tools of Government qui constitue l'une des caractéristiques de la nouvelle gouvernance publique ${ }^{103}$. L'approche fondée sur «les outils de gouvernance» correspond à une diversification de l'action publique qui a fait

92. Secrétariat du Conseil du Trésor du canada, «Directive du Cabinet sur la rationalisation de la réglementation», Ottawa, 2007, en ligne: http:// publications.gc.ca/site/fra/309288/publication.html.

93. Secrétariat du Conseil du Trésor du Canada, «Directive du Cabinet relative à la production d'une analyse de l'impact socio-économique de la réglementation", in Manuel de la politique administrative, Ottawa, Approvisionnement et Services Canada, 1978, chap. 490, appendice E.

94. Sur cette évolution, voir F. Houle, Analyses d'impact et consultations réglementaires au Canada. Étude sur les transformations du processus réglementaire fédéral: de la réglementation pathogène à la réglementation intelligente, Montréal, Y. Blais, 2012, p. 227.

95. Statutory Instruments Act/ loi sur les textes réglementaires, LRC 1985, chap. S-22.

96. Projet de loi C-25, loi sur les règlements, $2^{\mathrm{e}}$ session, $35^{\mathrm{e}}$ législature, 42-43 Elisabeth II, en ligne: https://www.parl.ca/DocumentViewer/fr/35-1/ projet-loi/C-84/premiere-lecture (projet de loi C-84, remplacé à l'identique par le projet de loi C-25).

97. P. Issalys, «L'analyse d'impact des lois et règlements: impératif d'efficacité ou condition de légitimité ?», in Interpretatio non cessat. Mélanges en l'honneur de Pierre-André Côté, S. Beaulac, M. Devinat (dir.), Montréal, Y. Blais, 2011, p. 133-141.

98. OCDE, Politique de la réglementation: vers un nouvel agenda (conférence de l'OCDE sur la politique de la réglementation, Paris, 28-29 octobre 2010), p. 25, en ligne: https://www.oecd.org/fr/gov/politique-reglementaire/47747059.pdf.

99. Secrétariat du Conseil du Trésor du Canada, «Directive du Cabinet sur la réglementation», art. 5.2.2 et 5.2.3.

100. Ibid., art. 5.1.2 («Choix de l'instrument»).

101. Ibid.

102. Secrétariat du Conseil du Trésor du Canada, «Politique sur l'élaboration de la réglementation», art. 7.1.3 («Sélection de l’instrument»).

103. The Tools of Government: A Guide to the New Governance, L. M. Salomon (dir.), Oxford, Oxford University Press, 2002. 
l'objet de plusieurs travaux au Canada ${ }^{104}$ et en France ${ }^{105}$. L'insistance sur les solutions de rechange à la réglementation vise à promouvoir la combinaison de divers types d'instruments, classiques ou moins traditionnels, afin de raffiner davantage les procédés de l'action publique. L'approche réglementaire classique perd ainsi son exclusivité au profit du «choix des instruments d'intervention ${ }^{106}$. Cette relativisation de la réglementation a suscité des travaux sur «l'infléchissement des formes juridiques de l'action étatique ${ }^{107}$. Afin de rompre avec une rationalité strictement comptable et économique, Pierre Issalys a proposé une vision plus contemporaine des critères de justification des interventions publiques: la maîtrise politique, la qualité de l'espace public à des fins de participation et de débat, l'équité à des fins procédurales, la compétence technique fondée sur l'expertise, et enfin, l'économie de moyens afin de minimiser les coûts ${ }^{108}$. Dans la «Politique sur l'élaboration de la réglementation» (art. 7.1.3), les autorités fédérales privilégient l'efficacité technique dans l'atteinte des objectifs, le principe de précaution (le degré et le type de risque), la diminution du fardeau réglementaire pour les petites entreprises, et surtout, la méthode de type coûts / avantages.

La volonté de cantonnement de la réglementation n'en reste pas moins prépondérante dans le contexte de l'utilisation de la règle du « un pour un ${ }^{109}$. Déjà énoncée dans la version de 2012 de la directive du Cabinet sur la réglementation ${ }^{110}$, cette règle a été transposée dans la loi de 2015 sur la réduction de la paperasse ${ }^{111}$. Cette règle $\mathrm{du}$ «one-for-one», d'inspiration britannique, est ainsi formulée à l'article 5 :

Lorsque la prise d'un règlement impose un nouveau fardeau administratif aux entreprises, le coût de ce fardeau doit être compensé par la modification ou l'abrogation d'un ou plusieurs règlements.

Cette approche de freinage et de limitation de l'action réglementaire se traduit également par la volonté d'intégrer par renvoi des règles techniques et scientifiques. Connu en anglais sous le terme "incorporation by reference», le procédé du renvoi a souvent été traduit de façon littérale au Canada ${ }^{112}$. Ce procédé de substitution est en pleine expansion sans que cette évolution soit très visible. Sur une base ponctuelle, c'est le législateur qui autorise le renvoi à des règles techniques. Dans le défunt projet de loi C-25 (1995), les autorités fédérales avaient formulé quatre dispositions précises afin d'introduire des balises en matière de renvoi ${ }^{113}$. L'abandon de ce projet de loi ne permettait plus d'offrir des repères suffisants pour les organismes et ministères fédéraux. Si l'utilisation de ces «normes, lignes directrices et recommandations nationales ou internationales » est esquissée dans la directive de $2012^{114}$, la dernière version de 2018 prévoit le principe de «l'incorporation par renvoi ${ }^{115}$. C'est néanmoins la «Politique sur l'élaboration de la réglementation» $(2018$, art. 5$)$ qui offre un cadre plus précis. Les documents incorporés par renvoi sont intégrés au règlement afin d'être "juridiquement contraignants". Dans le résumé de l'étude d'impact de la réglementation (REIR), l'organisme de réglementation doit préciser si le renvoi est de nature statique ou dynamique, si les documents référents sont accessibles et disponibles dans les deux langues officielles ${ }^{116}$, et si les obligations commerciales internationales du Canada sont respectées. La tendance lourde est à l'harmonisation puisque

Les organismes de réglementation doivent d'abord considérer [...] l'adoption et l'incorporation d'une norme internationale existante avant de considérer l'élaboration d'une norme canadienne unique ${ }^{117}$.

En dépit de la loi de 2015 sur l'élimination de la paperasse, les transformations les plus significatives ont été introduites par des directives, des lignes directrices, des politiques et des manuels. Les autorités fédérales n'ont pas jugé opportun de modifier la loi sur les textes réglementaires afin d'introduire le mécanisme de l'étude d'impact, ainsi que celui de l'incorporation par renvoi.

104. Designing Government: From Instruments to Governance, P. F. Eliadis, M. M. Hill, M. Howlett (dir.), Montréal, McGill-Queen's University Press, 2005; W. A. Bogart, "The Tools of the Administrative State and the Regulatory Mix», in Administrative Law in Context, C. M. Flood,

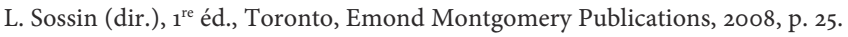

105. Gouverner par les instruments, P. Lascoumes, P. Le Galès (dir.), Paris, Les Presses de Sciences Po, 2005; L'instrumentation de l'action publique, C. Halpern, P. Lascoumes, P. Le Galès (dir.), Paris, Les Presses de Sciences Po, 2014.

106. M. J. Trebilcock et al., Le choix des instruments d'intervention, Ottawa, ministre des Approvisionnements et Services Canada, 1982.

107. P. Issalys, Répartir les normes. Le choix entre les formes d'action étatique, Québec, Société de l'assurance automobile du Québec, 2002 , p. 16.

108. Ibid., p. 308.

109. D. Mockle, «La réglementation intelligente...», p. 1235.

110. Secrétariat du Conseil du Trésor du Canada, «Directive du Cabinet sur la gestion de la réglementation », Ottawa, 2012, en ligne: https://www.canada.ca/ $\mathrm{fr} /$ secretariat-conseil-tresor/services/gestion-reglementation-federale/lignes-directrices-outils/directive-cabinet-gestion-reglementation.html.

111. Loi sur la réduction de la paperasse, LC 2015, chap. 12. Le titre complet est plus explicite: loi visant à limiter le fardeau administratif que la réglementation impose aux entreprises. L'expression « réduction de la paperasse» n'est pas d'origine canadienne (OCDE, Éliminer la paperasserie. Des stratégies nationales pour simplifier les formalités administratives, Paris, Publications de l'OCDE, 2006). Le titre anglais est mieux réussi: Red Tape Reduction Act. Il aurait été plus approprié et plus élégant de faire état d'une loi de simplification administrative.

112. J. Desjardins, J. Legault, «L'incorporation par renvoi dans l'exercice du pouvoir réglementaire à l'échelon fédéral», Revue du Barreau canadien, no 70,1991, p. 244

113. Projet de loi C-25, art. 16-19.

114. Secrétariat du Conseil du Trésor du Canada, «Directive du Cabinet sur la gestion de la réglementation», art. 26 (ix).

115. Secrétariat du Conseil du Trésor du Canada, «Directive du Cabinet sur la réglementation», art. 5.2.6.

116. Pour les documents unilingues, des justifications sont requises et des lignes directrices doivent être élaborées.

117. Secrétariat du Conseil du Trésor du Canada, «Politique sur l'élaboration de la réglementation», art. 7.1.4 («Incorporation par renvoi»). 
Même si la mise en œuvre de ces mécanismes requiert des règles explicatives pour les organismes et ministères fédéraux, les autorités fédérales ne peuvent pas modifier le cadre législatif qui régit l'action réglementaire par des directives et des politiques. Il faut modifier ou remplacer la loi sur les textes réglementaires de façon à introduire le principe de l'étude d'impact, ainsi que le mécanisme de l'incorporation par renvoi. Le Québec ne fait guère mieux en ayant élaboré une politique gouvernementale ${ }^{118}$ qui modifie en substance la loi sur les règlements ${ }^{119}$.

\section{La nécessité d'un cadre législatif}

Entre les exigences de motivation requises pour des actes issus de l'exercice d'une fonction administrative ou juridictionnelle, et celles attendues pour la justification des actes réglementaires, il n'est pas exagéré de conclure que le Canada est de plus en plus imprégné par cette culture de justification qui caractérise l'évolution de plusieurs systèmes nationaux de droit public ${ }^{120}$. Les enjeux ne sont toutefois pas du même type entre les actes individuels et ceux de portée générale, puisque les exigences requises pour les premiers visent à fonder, sur une base rationnelle, la recherche des motifs appropriés en droit et en fait. Pour les seconds, la distanciation par rapport au droit formel est beaucoup plus affirmée puisque les autorités cherchent non seulement à justifier la pertinence scientifique, économique, sociale et technique de la réglementation, mais valorisent la recherche de solutions de rechange à la réglementation classique sous forme de règlements. Le phénomène contemporain de la réglementation intelligente contribue de façon singulière à transposer sur une tout autre échelle ces exigences de justification.

Malgré ces différences, la démarche canadienne se caractérise par une sous-utilisation de la loi. Cette tendance est très nette en droit fédéral où aucune loi de portée générale n'a été élaborée en matière de procédure administrative, tant pour la procédure administrative non contentieuse, que pour celle susceptible de baliser le fonctionnement des tribunaux administratifs. Pour la mise en œuvre de la réglementation intelligente, le même désintérêt est manifeste. Ce manque d'ancrage législatif et constitutionnel offre un net contraste avec l'évolution récente de la France où l'outil législatif reste très valorisé pour la motivation des actes administratifs individuels (Code des relations entre le public et l'administration), mais également la recherche d'une dimension constitutionnelle. Ainsi, l'obligation d'étude d'impact des projets de loi repose sur un fondement constitutionnel depuis $2008^{121}$. Sur ce plan, la France n'est pas un cas isolé. Le contraste entre l'approche européenne et la «frilosité» de la démarche canadienne a été remarqué ${ }^{122}$.

Cette différence culturelle et politique est également tangible pour d'autres enjeux liés à la motivation et à la transparence. Dans un contexte marqué par l'utilisation croissante de l'intelligence artificielle au sein de l'administration fédérale, le Secrétariat du Conseil du Trésor a rendu publique une nouvelle «Directive sur la prise de décision automatisée ${ }^{123}$. À des fins de transparence, elle oblige les ministères et organismes fédéraux à afficher sur leur site Internet des avis afin d'indiquer que des décisions puissent être rendues totalement ou en partie par un système décisionnel automatisé à la lumière de plusieurs types d'exigences énumérées en annexe ${ }^{124}$. Dans le même esprit, il est nécessaire de «fournir une explication significative aux personnes concernées sur la façon dont la décision a été prise et la raison pour laquelle elle a été prise ${ }^{125}$. Avant cette politique, les autorités fédérales n'avaient pas manifesté beaucoup d'intérêt pour la motivation des décisions administratives. L'appréhension des enjeux liés à l'automatisation de plusieurs processus décisionnels reste néanmoins cantonnée à des dimensions administratives et techniques. Si cette nouvelle politique offre en annexe les définitions de différents concepts (décision administrative, évaluation de l'incidence algorithmique, intelligence artificielle, système décisionnel automatisé, équité procédurale, code source), elle montre, en définitive, que les autorités fédérales sont surtout préoccupées par les conditions de mise en œuvre au détriment de la légitimité qui résulte de l'utilisation de la loi. Les enjeux juridiques sont subsumés dans ces considérations d'efficacité et de rationalisation technique. Sur ce plan, la comparaison avec la France montre une nette différence puisque deux dispositions $\mathrm{du}$ Code des relations entre le public et l'administration «prévoient deux procédés qui permettent la communication et la diffusion des principales caractéristiques des algorithmes fondant une décision individuelle $»^{126}$.

118. Gouvernement du Québec, «Politique gouvernementale sur l'allègement réglementaire et administratif - Pour une réglementation intelligente», 2018, en ligne: https:/www.economie.gouv.qc.ca/fileadmin/contenu/publications/administratives/allegement/politique_gouv_allegement.pdf.

119. Loi sur les règlements, LRQ, chap. R-18.1.

120. La formule «ethos of justification " a été utilisée par la juge en chef B. McLachlin dans son texte "The Role of Administrative Tribunals and Courts in Maintening the Rule of Law», Canadian Journal of Administrative Law and Practice, n 12, 1999, p. 174.

121. B.-L. Combrade, L'obligation d'étude d'impact des projets de loi, Paris, Dalloz, 2017, p. 1.

122. P. Issalys, «L'analyse d'impact des lois et règlements...», p. 165

123. Secrétariat du Conseil du Trésor du Canada, «Directive sur la prise de décision automatisée», Ottawa, $1^{\mathrm{er}}$ avril 2019, en ligne: https://www. tbs-sct.gc.ca/pol/doc-fra.aspx?id=32592.

124. Ibid., art. 6.2.1.

125. Ibid., art. 6.2.3.

126. E. Mouriesse, «L'opacité des algorithmes et la transparence administrative», Revue française d'administration publique, 2019, p. 47. Il s'agit des articles L. 312-1-3 et L. 311-3-1 du Code des relations entre le public et l'administration. Les conditions fondant le traitement algorithmique d'une décision administrative peuvent être communiquées à l'intéressé s'il en fait la demande. Un décret en Conseil d'État est prévu pour l'application de l'article L. 311-3-1 
Cette volonté de gouverner par des directives, des politiques et des lignes directrices n'explique pas tout. Les études disponibles sur la thématique de la motivation montrent un certain degré de méprise à propos des finalités du contrôle judiciaire. Comme l'a rappelé à juste titre Madame la juge en chef McLachlin, le rôle premier des cours et des tribunaux est de faire respecter la primauté du droit (Rule of Law) ${ }^{127}$. Si ce contrôle a permis une avancée significative dans le cadre de l'arrêt Baker, c'est notamment parce que les motifs fondant la décision des agents d'immigration étaient disponibles. Si les motifs deviennent insuffisants ou incomplets, la tâche des juges devient plus difficile et le fardeau qui incombe au justiciable devient considérable. Le contrôle judiciaire reste un contrôle $a$ posteriori qui vise à assurer la validité et la légalité de l'action administrative. Dans cette perspective, la Cour suprême sera encore appelée à évaluer des litiges où des lacunes dans la motivation de décisions administratives ou juridictionnelles seront alléguées. Dans le cadre du contentieux constitutionnel, lorsque des atteintes à des droits protégés par les exigences de la justice fondamentale sont invoquées (article 7 de la Charte canadienne des droits et libertés), cette tâche sera plus facile afin de contrôler la validité des motifs. En revanche, dans le cadre du contentieux administratif qui a été commenté dans notre étude, cette tâche devient plus aléatoire en l'absence d'une obligation générale de motiver toutes les décisions défavorables.

Le caractère indispensable du contrôle judiciaire ne peut pas exempter les juristes de revoir plus attentivement les obligations des autorités administratives dans un contexte où prévalent les considérations de transparence et d'accessibilité dans le champ du droit administratif. Dans une perspective de célérité et d'accessibilité où «les droits fondamentaux des administrés » sont pris en compte, la loi sur la justice administrative permet au Tribunal administratif du Québec de requérir, suite à l'examen de la requête et de la décision contestée, de la part de l'organe concerné qu'il prenne position sur certaines questions lorsqu'il a omis de le faire alors que la loi l'y obligeait ${ }^{128}$. Cette disposition complète l'article 8 qui oblige la motivation des décisions défavorables ${ }^{129}$. Cette approche montre la nécessité de la loi afin d'assurer de meilleures conditions pour le respect des droits des citoyens, et de façon plus générale, ceux du public. Contrairement à des idées reçues, la souplesse et la flexibilité ne sont pas l'apanage du seul droit jurisprudentiel.

\title{
Liste des sigles désignant les recueils de lois dans les références en notes de bas de page
}

\author{
LC Lois du Canada \\ LRC Lois refondues du Canada \\ LQ Lois du Québec \\ LRQ Lois refondues du Québec \\ LO Lois de l'Ontario \\ LRO Lois refondues de l'Ontario \\ RCS Recueil des arrêts de la Cour suprême \\ RSA Revised Statutes of Alberta \\ RSS Revised Statutes of Saskatchewan \\ SA Statutes of Alberta \\ SBC Statutes of British Columbia
}

127. B. McLachlin, «The Role of Administrative Tribunals and Courts...». Voir également l'arrêt Dunsmuir, \$30 (contrôle judiciaire et primauté du droit).

128. Loi sur la justice administrative, art. 116.

129. «L'autorité administrative motive les décisions défavorables qu'elle prend et indique, le cas échéant, les recours autres que judiciaires prévus par la loi, ainsi que les délais de recours». 